\title{
(\#WomeninDDW) Persistent Speaker Gender Gap at the Premier Digestive Disease Event
}

\author{
Zubair Khan ${ }^{1} \cdot$ Rabia Rukhshan ${ }^{1} \cdot$ Asmeen Bhatt $^{1}$ - Sushovan Guha ${ }^{1}$ - Srinivas Ramireddy ${ }^{1} \cdot$ Prithvi Patil $^{1}$. \\ Ricardo Badillo ${ }^{1} \cdot$ Roy Tomas DaVee ${ }^{1} \cdot$ Nirav Thosani ${ }^{1}$ (1)
}

Received: 3 September 2020 / Accepted: 13 December 2021 / Published online: 30 January 2022

(c) The Author(s), under exclusive licence to Springer Science+Business Media, LLC, part of Springer Nature 2021

\begin{abstract}
Background Over the last few decades, advances have been made regarding gender equality starting from medical students to trainees, to leadership in academics. The female representation in specialty academic conferences not only reflects the existing gender disparities in that specialty but also can influence young female trainees to join that field. Digestive Disease Week (DDW) is the premier digestive disease event. We aimed to calculate the proportion of female representation among speakers and moderators at the DDW meetings held from 2018 to 2020.

Methods The data for DDW 2018-2020 were collected via the online web-based planner. The gender of speakers of presentations and moderators of sessions were identified by a google search. We further categorized the data by each participating society (AGA, ASGE, AASLD, and SSAT), by presentation track, by session track, and total overall representation in each year.

Results Despite the subject of the gender gap being in focus, the proportion of female moderators and speakers was low in DDW in the last 3 years. The female speakers constituted 31.6\% in 2018, 33.8\% in 2019 and $34.6 \%$ in 2020. There was slightly improved female representation in sessions of Inflammatory Bowel Disease, Stomach, and Small Bowel Disorders, Microbiome in GI \& Liver disease, and Basic Science over the last 3 years.

Conclusion Based on our study and those referenced in this article, we believe that strategies to promote the inclusivity of female moderators and speakers at DDW provide a huge opportunity to influence gender equity within GI.
\end{abstract}

Keywords Gender disparity · Speaker · Gastroenterology · Conferences

\section{Introduction}

Gender and racial disparities in medicine have been very well documented. Gender disparities have long existed at multiple levels starting from the number of female students

Nirav Thosani

Nirav.Thosani@uth.tmc.edu

Zubair Khan

Zubair.Khan@uth.tmc.edu

Rabia Rukhshan

rabiarukhshan29@gmail.com

Asmeen Bhatt

Asmeen.Bhatt@uth.tmc.edu

Sushovan Guha

Sushovan.Guha@uth.tmc.edu

Srinivas Ramireddy

Srinivas.Ramireddy@uth.tmc.edu joining medical schools to the number of female trainees enrolling in different specialties to the number of female trainees promoted to higher ranks in academic medicine in their respective institutions after being hired. Due to the continued focus on these disparities, a few advancements have

Prithvi Patil

Prithvi.B.Patil@uth.tmc.edu

Ricardo Badillo

Ricardo.Badillo@uth.tmc.edu

Roy Tomas DaVee

Roy.T.DaVee@uth.tmc.edu

Interventional Gastroenterology at the University of Texas (iGUT), McGovern Medical School at UTHealth, 6400

Fannin, Suite 1400, Houston, TX 77030, USA 
been made including a rising proportion of female medical students over recent years, from $46.9 \%$ in 2015 to $49.5 \%$ in 2018. In 2019, women comprised $50.5 \%$ of all medical school students [1]. Some of the contributors to gender disparities are universal and are not limited to the field of medicine alone; including a persistent pay gap, policies that discriminate against new and expecting mothers, and overt sexual harassment [2]. According to the Association of American Medical Colleges (AAMC) data from 2017, $17.6 \%$ of all practicing gastroenterologists are women. [3]. As noted in a previous editorial from 2006 [4], the percentage of female trainees entering gastroenterology (GI) doubled between 2000 and 2004 and as of 2018, 39\% of firstyear gastroenterology fellows in the nation are females [5].

Like other procedural specialties, GI is a demanding field with unpredictable duty hours, arduous inpatient services, and busy after-hour calls with life-threatening emergencies. Female gastroenterologists who must balance their professional and personal lives because of their expected societal roles at home are more prone to leave academic medicine $[6,7]$. Likewise, those who remain in academics must strive more to achieve the same as their male counterparts. Higher female representation is an important indicator of gender equity and serves as a source of improved career satisfaction and retention in academics. The higher representation of females in academic conferences besides an opportunity for career growth serves as a projection of more role models for female trainees and subsequently influences them to pursue academics.

A large study of 181 medical conferences held in North America over a decade found an increase in the proportion of female speakers from 25 to 34\% [8]. Digestive Disease Week ${ }^{\circledR}(\mathrm{DDW})$ is the world's largest gathering of physicians, researchers, and industry in the fields of GI, hepatology, endoscopy, and gastrointestinal surgery with over 14,000 attendees and 5400 original lectures, posters, and oral abstract presentations [9]. The purpose of our study was to identify the proportion of female representation among speakers and moderators at the DDW meetings held from 2018 to 2020 to explore the speaker gender gap in this largest gastroenterology meeting and identify the sessions, societies, and areas where this gap is more evident. This is the first study analyzing DDW data for speaker and moderator gender gaps. DDW is a premier event co-sponsored by four societies-American Gastroenterological Association (AGA), American Association for Gastrointestinal Endoscopy (ASGE), American Association for the Study of Liver Diseases (AASLD), and The Society for Surgery of the Alimentary Tract (SSAT).

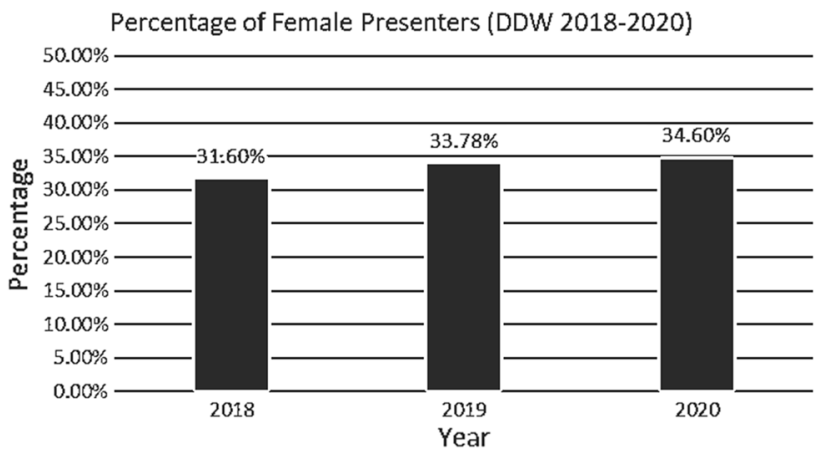

Fig. 1 Percentage of female speakers/presenters DDW 2018-2020

\section{Methods}

The DDW abstracts are available for the meeting attendees via online planners. These planners are available in both the web and mobile application versions. The abstracts are also published in the supplemental issues of the official journals of the respective societies organizing the event. The data for DDW 2018 and 2019 were collected via the online web-based planner. The DDW 2020 meeting was canceled because of an ongoing global pandemic of COVID-19 and the authors and speakers were given the option of uploading e-posters and recorded presentations for the selected oral abstracts and presentations [10]. We determined the proportion of women speakers selected for the oral presentation of abstracts and presentations. Two authors (Z.K \& R.R) identified the gender of speakers of abstracts and presentations by google search with $100 \%$ concordance of gender identity data. The moderators of sessions were identified similarly. Individual speakers could be counted multiple times as the main objective was to calculate the proportion of female speakers in total accepted abstracts and presentations with few speakers having more than one accepted abstract and presentation. We further categorized the data by each participating society (AGA, ASGE, AASLD, and SSAT), by presentation track, by session track and total overall representation in each year. The abstracts that were not assigned to any of the four societies in the 2018 and 2019 meetings were categorized under DDW which was not the format used in 2020. While both speaker and moderator data were available for DDW 2018 \& 2019, only presenter data were available for DDW 2020 because of the change in the format of the meeting. The data for session type were also not available in DDW 2020. 


\section{Results}

Overall, the proportion of female moderators and speakers was $31.22 \%(n=212) \& 31.60 \%(n=615)$, respectively, in 2018 (Fig. 1). In 2019, there was no significant improvement in trend with these percentages being $30.45 \%$ $(n=212) \& 33.78 \%(n=755)$, respectively (Fig. 1). In 2020 , the female presenters increased to $34.60 \%(n=3178)$ of the total presenters (Fig. 1 and Table 1). There was a slightly higher representation of female presenters in Distinguished Abstract Plenary ( $n=20,41.6 \%$ compared to $n=26,48.1 \%$ in 2019), Special Sessions ( $n=4,100 \%$ compared to $n=39,41.48 \%$ in 2019), Quick Shot $(n=26$, $36.6 \%$ compared to $n=53,44.91 \%$ in 2019) and Video Sessions ( $n=7,38.8 \%$ compared to $n=23,41.07 \%$ in 2019) (Table 1). The female presenters in ASGE Society sessions remained the same [2018: $n=122(26.87 \%)$ vs. 2019: $n=117(28.67 \%)]$ and that in AGA [2018: $n=354$ (33.9\%), 2019: $n=430$ (33.75\%), 2020: $\mathrm{n}=1940$ (38.38\%)] and SSAT [2018: $n=63$ (31.81\%), 2019: $n=95$ (37.54\%), 2020: $n=395$ (33.22\%)] improved over the last 3 years (Table 1). The female representation in AASLD sessions dropped from $2019[n=53(41.08 \%)]$ to 2020 $[n=382(36.73 \%)]$. There was improved female representation in sessions of Inflammatory Bowel Disease [2018: $n=57$ (31.66\%), 2019: $n=43$ (30.71\%), 2020: $n=447$ (38.90\%)], Stomach and Small Bowel Disorders [2018: $n=36$ (34.61\%), 2019: $n=41$ (34.74\%), 2020: $n=120$ (39.21\%)], Microbiome in GI \& Liver disease [2018: $n=38$ (41.30\%), 2019: $n=37$ (43.52\%), 2020: $n=238$ (43.19\%)], and Basic Science [2018: $n=81(39.90 \%)$, 2019: $n=145$ (44.20\%), 2020: $n=449$ (41.07\%)] over the last 3 years (Table 1).

\section{Discussion}

The overall mean proportion of female speakers and moderators at DDW in the past 3 years was quite low, which could in part be attributed to the overall gender gap in GI in North America with $82.4 \%$ of practicing gastroenterologists being male [3]. As explained in the results, most of the speakers of the accepted abstracts and presentations were from the USA, the gender gap was even more pronounced in speakers outside the USA, with female speakers constituting only one-sixth of the total presenters. A large international coordinated effort is needed to encourage females to opt for the field of gastroenterology and to provide them with better opportunities of worklife balance for demands of family life. Similarly, female trainees also constituted one-sixth of total presentations though they constitute one-third of total gastroenterology trainees in the USA. The obligations of family life and children may preclude them from traveling and presenting in conferences. Providing young females, the facilities like daycare and family corners for children needs like breastfeeding may facilitate and encourage their participation in the meetings. There was a slightly better representation of females in the presenter category of the basic science track. The AAMC also has shown that there is a greater proportion of female authorship in basic/translational science research when compared to clinical research. [11].

The topic of the speaker gender gap in medical conferences gives a perspective into overall gender inequality of gender disparity in a particular field. Various gastroenterology societies like ASGE, ACG, and AGA have taken some steps as outlined below to address the issue. A study that aimed to characterize female representation among faculty in courses sponsored by the ASGE found that between 2009 and 2014 women constituted $19 \%$ of the faculty positions and that female faculty participation exceeded the ASGE female domestic membership rate in all years. Women were more likely to serve as course directors than lecturers and to participate in smaller courses [12]. Since then, ASGE launched the Women in GI Small Interest Group (SIG) in 2019 [13] to promote the networking and advancement of its over $1700+$ female members. Also, in April 2019, two other societies American College of Gastroenterology (ACG) and the North American Society for Pediatric Gastroenterology, Hepatology and Nutrition (NASPGHAN) launched the \#DiversityinGI social media campaign, by increasing the visibility of women in GI and promoting diversity and inclusion in GI for both adult and pediatric practitioners [14].

In February 2019, the renowned medical journal The Lancet published a special edition of the journal dedicated to advance women in science, medicine, and health and the editors of The Lancet group of journals made a public commitment to increasing the representation of women and colleagues from low-income and middle-income countries among their editorial advisers, peer reviewers, and authors [15]. On July 6, 2020, AGA Governing Board announced the creation of the AGA Equity Project where the task force will develop a multi-year strategic plan to achieve several aims including the one that will focus on building a diverse member group with women included in the organizational decision-making process [16]. A recent analysis of the visibility of women at the American College of Gastroenterology (ACG) annual meeting showed that the proportion of women serving as Post-Graduate Course faculty increased from 11.7 to $30.5 \%$ and Annual Scientific Meeting faculty from 14.1 to $45.4 \%$ between 2010 and 2019 [17]. 
Table 1 Statistics of female speakers and moderators in DDW meetings (2018-2020)

\begin{tabular}{|c|c|c|c|}
\hline Year & 2018 & 2019 & 2020 \\
\hline \multicolumn{4}{|l|}{ Total } \\
\hline Female moderators $N(\%$ age $)$ & $212(31.22 \%)$ & $212(30.45 \%)$ & N/A \\
\hline Female speakers $N(\%$ age $)$ & $615(31.60 \%)$ & $755(33.78 \%)$ & $3178(34.6 \%)$ \\
\hline \multicolumn{4}{|l|}{ Breakout by session type } \\
\hline \multicolumn{4}{|l|}{ Clinical symposiums } \\
\hline Female moderators $N(\%$ age $)$ & $64(31.06 \%)$ & $61(30.04 \%)$ & N/A \\
\hline Female speakers $N(\%$ age $)$ & $142(27.51 \%)$ & $150(27.7 \%)$ & \\
\hline \multicolumn{4}{|l|}{ Committee sponsored symposium } \\
\hline Female moderators $N(\%$ age $)$ & $21(45.65 \%)$ & $20(40.81 \%)$ & N/A \\
\hline Female speakers $N(\%$ age $)$ & $42(34.71 \%)$ & $67(40.11 \%)$ & \\
\hline \multicolumn{4}{|l|}{ Conference/debate } \\
\hline Female moderators $N(\%$ age $)$ & $4(25 \%)$ & $3(15.78 \%)$ & N/A \\
\hline Female speakers $N(\%$ age $)$ & $9(14.51 \%)$ & $12(22.64 \%)$ & \\
\hline \multicolumn{4}{|l|}{ Distinguished abstract plenary } \\
\hline Female moderators $N(\%$ age $)$ & $5(35.71 \%)$ & $2(14.28 \%)$ & N/A \\
\hline Female speakers $N(\%$ age $)$ & $20(41.66 \%)$ & $26(48.14 \%)$ & \\
\hline \multicolumn{4}{|l|}{ Plenary session } \\
\hline Female Moderators $N(\%$ age $)$ & $16(39.02 \%)$ & $3(16.66 \%)$ & N/A \\
\hline Female Speakers $N(\%$ age $)$ & $45(34.88 \%)$ & $24(33.80 \%)$ & \\
\hline \multicolumn{4}{|l|}{ Quick shot } \\
\hline Female moderators $N(\%$ age $)$ & $2(16.66 \%)$ & $5(27.77 \%)$ & N/A \\
\hline Female speakers $N(\%$ age $)$ & $26(36.61 \%)$ & $53(44.91 \%)$ & \\
\hline \multicolumn{4}{|l|}{ Research forum } \\
\hline Female moderators $N(\%$ age $)$ & $67(31.60 \%)$ & $68(33.17 \%)$ & N/A \\
\hline Female speakers $N(\%$ age $)$ & $228(35.34 \%)$ & $245(36.51 \%)$ & \\
\hline \multicolumn{4}{|l|}{ Research symposium } \\
\hline Female moderators $N(\%$ age $)$ & $10(29.41 \%)$ & $14(37.83 \%)$ & N/A \\
\hline Female speakers $N(\%$ age $)$ & $28(38.88 \%)$ & $25(29.41 \%)$ & \\
\hline \multicolumn{4}{|l|}{ Roundtable with experts } \\
\hline Female moderators $N(\%$ age $)$ & - & - & N/A \\
\hline Female speakers $N(\%$ age $)$ & $0(0 \%)$ & $7(38.88 \%)$ & \\
\hline \multicolumn{4}{|l|}{ Special session } \\
\hline Female moderators $N(\%$ age $)$ & $2(100 \%)$ & $7(43.75 \%)$ & N/A \\
\hline Female speakers $N(\%$ age $)$ & $4(100 \%)$ & $39(41.48 \%)$ & \\
\hline \multicolumn{4}{|l|}{ State of art lecture } \\
\hline Female moderators $N(\%$ age $)$ & $3(50 \%)$ & $1(16.66 \%)$ & N/A \\
\hline Female speakers $N(\%$ age $)$ & $4(33.33 \%)$ & $4(28.57 \%)$ & \\
\hline \multicolumn{4}{|l|}{ Topic forum } \\
\hline Female moderators $N(\%$ age $)$ & $9(15.51 \%)$ & $9(18 \%)$ & N/A \\
\hline Female speakers $N(\%$ age $)$ & $37(21.14 \%)$ & $48(29.26 \%)$ & \\
\hline \multicolumn{4}{|l|}{ Translational symposium } \\
\hline Female moderators $N(\%$ age $)$ & $7(43.75 \%)$ & $12(27.90 \%)$ & N/A \\
\hline Female speakers $N(\%$ age $)$ & $7(25 \%)$ & $16(18.18 \%)$ & \\
\hline \multicolumn{4}{|l|}{ Video session } \\
\hline Female moderators $N(\%$ age $)$ & $1(16.66 \%)$ & $1(16.66 \%)$ & N/A \\
\hline Female speakers $N(\%$ age $)$ & $7(38.88 \%)$ & $23(41.07 \%)$ & \\
\hline \multicolumn{4}{|l|}{ Breakout by societies } \\
\hline \multicolumn{4}{|l|}{ AASLD } \\
\hline Female moderators $N(\%$ age $)$ & $18(33.33 \%)$ & $15(34.09 \%)$ & N/A \\
\hline Female speakers $N(\%$ age $)$ & $47(31.33 \%)$ & $53(41.08 \%)$ & $382(36.73 \%)$ \\
\hline
\end{tabular}


Table 1 (continued)

\begin{tabular}{|c|c|c|c|}
\hline Year & 2018 & 2019 & 2020 \\
\hline \multicolumn{4}{|l|}{ AGA } \\
\hline Female moderators $N(\%$ age $)$ & $135(34.17 \%)$ & $133(31.22 \%)$ & N/A \\
\hline Female speakers $N(\%$ age $)$ & $354(33.90 \%)$ & $430(33.75 \%)$ & $1940(38.38 \%)$ \\
\hline \multicolumn{4}{|l|}{ ASGE } \\
\hline Female moderators $N(\%$ age $)$ & $39(26.89 \%)$ & $40(29.85 \%)$ & N/A \\
\hline Female speakers $N(\%$ age $)$ & $122(26.87 \%)$ & $117(28.67 \%)$ & $461(24.31 \%)$ \\
\hline \multicolumn{4}{|l|}{ DDW } \\
\hline Female moderators $N(\%$ age $)$ & $10(27.02 \%)$ & $12(36.36 \%)$ & N/A \\
\hline Female Speakers $N(\%$ age $)$ & $29(29.29 \%)$ & $60(35.08 \%)$ & N/A \\
\hline \multicolumn{4}{|l|}{ SSAT } \\
\hline Female moderators $N(\%$ age $)$ & $10(20.83 \%)$ & $12(20.33 \%)$ & N/A \\
\hline Female speakers $N(\%$ age $)$ & $63(31.81 \%)$ & $95(37.54 \%)$ & $395(33.22 \%)$ \\
\hline \multicolumn{4}{|l|}{ Breakout by track } \\
\hline \multicolumn{4}{|l|}{ Basic science } \\
\hline Female moderators $N(\%$ age $)$ & $25(38.46 \%)$ & $34(33.66 \%)$ & N/A \\
\hline Female speakers $N(\%$ age $)$ & $81(39.90 \%)$ & $145(44.20 \%)$ & $449(41.07 \%)$ \\
\hline \multicolumn{4}{|l|}{ Biliary tract disease, } \\
\hline Female moderators $N(\%$ age $)$ & $5(17.85 \%)$ & $7(19.44 \%)$ & N/A \\
\hline Female speakers $N(\%$ age $)$ & $27(28.72 \%)$ & $31(28.70 \%)$ & $176(34.50 \%)$ \\
\hline \multicolumn{4}{|l|}{ Clinical practice } \\
\hline Female moderators $N(\%$ age $)$ & $18(34.61 \%)$ & $41(25.15 \%)$ & N/A \\
\hline Female speakers $N(\%$ age $)$ & $36(23.84 \%)$ & $133(26.70 \%)$ & $443(34.05 \%)$ \\
\hline \multicolumn{4}{|l|}{ Colorectal diseases } \\
\hline Female moderators $N(\%$ age $)$ & $20(32.78 \%)$ & $10(23.25 \%)$ & N/A \\
\hline Female speakers $N(\%$ age $)$ & $59(32.24 \%)$ & $63(32.47 \%)$ & $200(31.29 \%)$ \\
\hline \multicolumn{4}{|l|}{ Education and training } \\
\hline Female moderators $N(\%$ age $)$ & $15(42.85 \%)$ & $13(40.62 \%)$ & N/A \\
\hline Female speakers $N(\%$ age $)$ & $37(38.54 \%)$ & $41(41.00 \%)$ & $49(29.51 \%)$ \\
\hline \multicolumn{4}{|l|}{ Esophageal diseases } \\
\hline Female moderators $N(\%$ age $)$ & $12(30 \%)$ & $9(20.93 \%)$ & N/A \\
\hline Female speakers $N(\%$ age $)$ & $38(29 \%)$ & $54(32.53 \%)$ & $280(32.00 \%)$ \\
\hline \multicolumn{4}{|l|}{ Functional GI \& motility disorders } \\
\hline Female moderators $N(\%$ age $)$ & $7(26.92 \%)$ & $7(41.17 \%)$ & N/A \\
\hline Female speakers $N(\%$ age $)$ & $30(44.11 \%)$ & $18(33.96 \%)$ & $106(28.80 \%)$ \\
\hline \multicolumn{4}{|l|}{ Healthcare \& disparities } \\
\hline Female moderators $N(\%$ age $)$ & $10(41.66 \%)$ & $9(50 \%)$ & N/A \\
\hline Female speakers $N(\%$ age $)$ & $16(29.09 \%)$ & $19(38.77 \%)$ & $3(16.66 \%)$ \\
\hline \multicolumn{4}{|l|}{ Liver disease \& transplantation } \\
\hline Female moderators $N(\%$ age $)$ & $19(30.64 \%)$ & $14(34.14 \%)$ & N/A \\
\hline Female speakers $N(\%$ age $)$ & $53(31.54 \%)$ & $38(35.18 \%)$ & $207(34.44 \%)$ \\
\hline \multicolumn{4}{|l|}{ Inflammatory bowel diseases } \\
\hline Female moderators $N$ (\% age) & $21(33.33 \%)$ & $16(42.10 \%)$ & N/A \\
\hline Female speakers $N(\%$ age $)$ & $57(31.66 \%)$ & $43(30.71 \%)$ & $447(38.90 \%)$ \\
\hline \multicolumn{4}{|l|}{ Microbiome in GI \& liver diseases } \\
\hline Female moderators $N$ (\% age) & $19(48.71 \%)$ & $13(40.62 \%)$ & N/A \\
\hline Female speakers $N(\%$ age $)$ & $38(41.30 \%)$ & $37(43.52 \%)$ & $238(43.19 \%)$ \\
\hline \multicolumn{4}{|l|}{ Obesity \& nutrition } \\
\hline Female moderators $N(\%$ age $)$ & $5(23.80 \%)$ & $6(35.29 \%)$ & N/A \\
\hline Female speakers $N(\%$ age $)$ & $16(32 \%)$ & $18(40.00 \%)$ & $114(36.65 \%)$ \\
\hline \multicolumn{4}{|l|}{ Pancreatic disease } \\
\hline Female moderators $N(\%$ age $)$ & $12(35.29 \%)$ & $11(42.30 \%)$ & N/A \\
\hline
\end{tabular}


Table 1 (continued)

\begin{tabular}{llll}
\hline Year & 2018 & 2019 & 2020 \\
\hline $\begin{array}{l}\text { Female speakers } N(\% \text { age }) \\
\text { Pediatric GI }\end{array}$ & $22(23.15 \%)$ & $19(24.67 \%)$ & $82(28.08 \%)$ \\
$\quad$ Female moderators $N(\%$ age $)$ & $4(40 \%)$ & $6(33.33 \%)$ & N/A \\
Female speakers $N(\%$ age $)$ & $10(41.66 \%)$ & $20(42.55 \%)$ & $35(33.33 \%)$ \\
Practice management & & & \\
Female moderators $N(\%$ age $)$ & - & $1(16.66 \%)$ & N/A \\
Female speakers $N(\%$ age $)$ & $5(13.51 \%)$ & $12(0 \%)$ & $18(18.55 \%)$ \\
Stomach and small bowel disorders & $36(34.61 \%)$ & $41(34.74 \%)$ & N/A \\
Female moderators $N(\%$ age $)$ & $18(22.78 \%)$ & $3(12 \%)$ & N/A \\
Female speakers $N(\%$ age $)$ & $56(23.43 \%)$ & $33(34.37 \%)$ & $211(26.47 \%)$ \\
Technologies \& procedural info & & & \\
Female moderators $N(\%$ age $)$ & & & \\
Female speakers $N(\%$ age $)$ & & & \\
\hline
\end{tabular}

\section{Limitations}

There are few limitations of this analysis. We only looked at a single conference, which is considered as one of the largest meetings in the field of gastroenterology. It would be interesting to look at other national and international conferences to assess the magnitude of gender gap in those meetings. We analyzed only the last 3 years' data, and it was interesting to note that despite the subject of the gender gap being in focus, no significant ground has been made in the premier digestive disease event. We did not include the first or presenting authors of the poster presentation for the gender gap in 2018 $\& 2019$ because of limitation of time and large data sets. We did analyze these data for the 2020 meeting as the format was changed to virtual. We did not analyze the other authors of abstracts apart from first or presenting authors to further explore gender equity as female authors are sometimes less likely to put themselves forward for speaking opportunities.

\section{Conclusions}

This is the first effort to characterize gender disparity in one of the premier digestive disease events in the world. A comprehensive review of a 3-year window (2018-2020) provides a snapshot of the status of gender inequality. Despite the focus on gender parity in medicine and its subspecialties, there remains a significant gender gap in the field of GI that needs to be addressed on multiple fronts. We propose few initiatives like selecting more females as organizers, including them in selection panels and Keynote speakers based on merit [18]. More female representation in the organizing committee is paired with female selection on panels and less likely to have all-male panels, which is criticized on social media like Twitter with \#manel. Individual societies should develop and sponsor mentoring programs for female trainees in research and academics to increase their productivity and selection in conferences as speakers. A recent study showed that interventions like increasing the number or visibility of female chairs or inviting a woman for an opening question in a session can improve female inclusion [19]. The speaker invitations are opportunities for personal and professional growth for the attendees and can increase their visibility in the field and inspire more younger trainees and attendees to pursue the field with the potential of mitigating the gender gap. DDW should publish gender, professional and academic profiles of the conference speakers, moderators, and panels to address any unconscious gender bias. Based on our study and those referenced in this article, we believe that strategies to promote the inclusivity of female moderators and speakers at DDW provide a huge opportunity to influence gender equity within GI.

Funding The study was partially funded from the Atilla Ertan MD endowment fund.

\section{Declarations}

Conflict of interest The authors disclose that there is no conflict of interest regarding the current study.

\section{References}

1. Total U.S. Medical School Enrollment by Race/Ethnicity (Alone) and Sex, 2015-2016 through 2019-2020. Available from: https:// www.aamc.org/system/files/2019-11/2019_FACTS_Table_B-3. pdf.

2. Krause ML, Elrashidi MY, Halvorsen AJ, et al. Impact of pregnancy and gender on internal medicine resident evaluations: a retrospective cohort study. J Gen Intern Med. 2017;32:648-653.

3. American Association of Medical Colleges Report of Active Physicians by Sex and Specialty. 2017; Available from: https://www. 
aamc.org/data-reports/workforce/interactive-data/active-physi cians-sex-and-specialty-2017.

4. Hecht, G., Women in gastroenterology: exciting times and trends, in Gastroenterology. 2008: United States. p. 913-4.

5. American Board of Internal Medicine (ABIM) Percentage of first-year fellows by gender and type of medical school attended. 2009-10 to 2018/19 Available from: https://www.abim.org/about/ statistics-data/resident-fellow-workforce-data/first-year-fellowsby-gender-type-of-medical-school-attended.aspx.

6. Nonnemaker L. Women physicians in academic medicine: new insights from cohort studies. N Engl J Med. 2000;342:399-405.

7. Kesavarapu K, Schwartz J, Ikonomi E, et al. What's holding women back? A review of gender inequality in gastroenterology in the USA. Lancet Gastroenterol Hepatol. 2019;4:898-900.

8. Ruzycki SM, Fletcher S, Madalene E, et al. Trends in the proportion of female speakers at medical conferences in the United States and in Canada, 2007-2017. JAMA Netw Open. 2019;2:e192103.

9. DDW (Digestive diseases week). Available from: https://ddw.org/.

10. DDW apprisor page. Available from: https://ddw.apprisor.org/ epsWelcome.cfm?CFID $=7399493 \&$ CFTOKEN $=$ bd743a05af 955e53-2BB7FE0D-BE39-E692-3971E21A4753DE04.

11. Association of American Medical Colleges. The 2018 physician specialty data report: active physicians by sex and specialty, 2017. Available at: https://urldefense.proofpoint.com/v2/url?u= https-3A__www.aamc.org_datareports_workforce_interactive2Ddata_active-2Dphysicians-2Dsex-2Dand-2Dspecialty-2D201 $7 \& d=D w I G a Q \& c=b K R y S V-o u E g \_A T-w 2 Q W s T d d 9 X \_K Y h 9 E$ q2fdmQDVZgw\&r=tdX1IuHLvwYvkMStZ0B_mJXIp8BMEz NctsSSYKjFVaE\&m=LbGf5XMv2pAMyCHB7WjdSbi5P1prLq kZte1W86avsck\&s=sMFyAHL_yKvqHjajlnm8VzerXdPcy8Fk5 ns0eSXLwAI\&e $=$
12. Enestvedt BK, DeVivo RS, Schmitt CM, et al. Increase in female faculty in American Society for Gastrointestinal Endoscopy-sponsored programming over time. Gastrointest Endosc. 2018;87:952-955.

13. Women in GI. Available from: https://www.asge.org/ women-in-gi-sig.

14. Diversity in GI ACGS. 2019; Available from: https://gi.org/2019/ 06/27/diversityingi-acgs-new-social-media-campaign/.

15. Advancing women in science, global medicine and global health. . 2019, The Lancet. p. 493-610.

16. AGA announces six point commitment to equity. Available from: https://gastro.org/news/aga-announces-six-point-commi tment-to-equity/.

17 Jansson-Knodell CL, Bhavsar-Burke I, Gayam S, et al. Visibility of women at the american college of gastroenterology annual meetings increasing over time. Am J Gastroenterol. 2021. https:// doi.org/10.14309/ajg.0000000000001345.

18. Rabinowitz LG, Anandasabapathy S, Sethi A, et al. Addressing gender in gastroenterology: opportunities for change. Gastrointest Endosc. 2020;91:155-161.

19 Salem V, McDonagh J, Avis E, et al. Scientific medical conferences can be easily modified to improve female inclusion: a prospective study. LANCET Diabetes Endocrinol. 2021. https://doi. org/10.1016/S2213-8587(21)00177-7.

Publisher's Note Springer Nature remains neutral with regard to jurisdictional claims in published maps and institutional affiliations. 